\title{
Nuove tendenze nei sistemi di controllo e di misurazione delle performance
}

\author{
Rosa Alba Miraglia
}

Il tema che lega tutti i contributi contenuti nel presente numero della rivista Management Control è la progettazione dei sistemi di controllo e di misurazione delle performance. In particolare, tali contributi mettono in evidenza la problematicità del rapporto tra scelte di gestione e sistema di controllo.

In questa sede, si osserva brevemente lo stato dell'arte del rapporto appena citato, si esaminano i riflessi che esso ha sulla progettazione del sistema di misurazione delle performance e si individuano le tendenze evolutive dei sistemi di controllo e di misurazione, desunte dall'analisi dei contributi pubblicati in questo numero.

\section{Rapporto tra scelte di gestione e sistema di controllo}

Come è noto, tradizionalmente la gestione d'impresa è distinta in due momenti, fra loro strettamente correlati: il momento strategico ed il momento operativo. Il primo centrato sulla creazione di vantaggi competitivi e quindi volto a comporre una serie interrelata di decisioni, in particolare quelle attinenti la "capacità". Tali decisioni, fortemente collegate alla strategia, hanno carattere episodico, producono effetti strutturali che vincolano la generale combinazione produttiva d'impresa e sono spesso irreversibili nel breve termine. Il momento operativo è invece centrato sull'impiego delle "capacità" allestite e disponibili. Le decisioni collegate a questo momento hanno effetti nel breve periodo, vengono assunte quotidianamente e da esse deriva lo svolgimento delle operazioni aziendali (acquisizione di fattori produttivi, produzione, ecc.).

$\mathrm{Ne}$ consegue che la gestione aziendale è fatta di decisioni (strategiche ed operative) e di operazioni, ma sostanzialmente sono queste ultime che portano ai risultati che provano o meno il raggiungimento della finalità aziendale consistente nell'equilibrio economico a valere nel tempo. Ne consegue anche che le decisioni strategiche influenzano le decisioni operative. Si può pertanto affermare che i caratteri della gestione sono definiti dal momento 
strategico, cioè dal momento creativo ed intuitivo; mentre il momento operativo ha rilevanza solo derivata, essendo esecutivo.

In questo contesto il sistema di controllo di gestione guida le operazioni della gestione corrente al fine di conseguire nel modo più efficace ed efficiente gli obiettivi che esprimono le linee strategiche. La letteratura, a causa della rilevanza delle decisioni strategiche, dedica attenzione alla comprensione del rapporto strategia/sistema di controllo di gestione. La strategia è un elemento esogeno al sistema di controllo; è imperniata su un "futuro" visto come estrapolazione del passato (la logica previsionale) ${ }^{1}$; è in linea con la "filosofia" aziendale rivolta alle dinamiche interne all'azienda e, quindi, all'efficienza quale variabile critica che consente di perseguire vantaggi di costo; è uno standard rispetto a cui riferire i risultati ed attivare il processo di feed-back (Anthony, 1967; Brunetti, 1979).

Ne consegue che sono i problemi che deve affrontare la gestione, sostanzialmente nel momento strategico, e le scelte ad essi connessi, che influenzano il sistema di controllo e richiedono interventi di progettazione o riprogettazione dello stesso sistema. In sintesi, il sistema di controllo è uno strumento che serve per implementare la strategia, dopo che la stessa è stata formulata. Esso, pertanto, riceve passivamente gli "ordini” dalla gestione strategica (Anthony, 1967; Andrews, 1971; Lorange, 1980; Eminente, 1986; Brusa, Zambrogna, 1991).

La tesi consolidata in letteratura mette quindi in evidenza il seguente nesso causale:

\section{scelte di gestione $\rightarrow$ sistema di controllo di gestione}

Questo nesso causale rimane invariato anche quando la gestione aziendale è posta di fronte a nuovi problemi legati all'instabilità ed incertezza dello scenario economico e all'aumento delle dimensioni aziendali. Ciò determina alcuni significativi cambiamenti nei caratteri della strategia. La strategia è imperniata su un "futuro" sul quale si possono formulare prospettive, cioè semplici indicazioni che non hanno carattere vincolante (logi-

\footnotetext{
${ }^{1}$ Ansoff et al., riportando i risultati di varie inchieste sulla programmazione, affermano: "Oggi, solo una manciata di aziende di primo piano usano la programmazione strategica per dirigere le loro spinte espansiva. Nella maggior parte dei casi si impiegano ancora le più semplici e primitive tecniche di programmazione a lungo termine basate sull'estrapolazione del passato e mancanti di quella sistematica elaborazione ed analisi delle alternative che caratterizzano la programmazione strategica”. Ansoff H.I., Declerck R.P., Hayes R.L. (1976), From Strategic Planning to Strategic Management, New York, Wiley \& Sons.
} 
ca prospettica) $)^{2}$; è in linea con la "filosofia" aziendale rivolta all'esterno, cioè verso il cliente, il mercato e l'ambiente esterno all'azienda. Ciò significa che, ai fini del vantaggio competitivo, bisogna prestare attenzione all'efficienza ma soprattutto all'efficacia, per ottimizzare il processo di generazione del valore, inteso come insieme dei risultati raggiunti con la gestione.

In questo contesto, il sistema di controllo di gestione non può limitarsi alla guida delle operazioni della gestione corrente, ma deve rivolgersi alla realizzazione sempre delle linee strategiche, che investono però aspetti ampli e complessi. Tale sistema rimane un semplice attuatore della strategia rispetto alla quale è del tutto estraneo. Ne consegue che la strategia influenza il sistema di controllo e richiede interventi di progettazione o riprogettazione dello stesso sistema. Permane quindi la linearità del rapporto tra scelte di gestione, sostanzialmente legate alla gestione strategica, e sistema di controllo di gestione. Tale linearità mal si concilia però con le attuali condizioni di forte instabilità ambientali, che non richiedono innanzitutto rigidità strategica.

Il modello strategico adottato, dove la strategia è un dato in genere non modificabile così come le relative scelte, non è adeguato ad un ambiente discontinuo. In quest'ultimo la strategia dovrebbe invece essere flessibile, al fine di adattarsi o meglio prevenire i segnali ambientali. La flessibilità strategica è un concetto da tempo ben noto negli studi di strategia. Mintzberg (1994), in particolare, richiama l'attenzione sul fatto che la strategia realizzata non coincide con quella deliberata.

Influenzati dagli studi di strategia, gli studi sul controllo si interessano non solo al controllo della gestione corrente ma anche al controllo della strategia. Si sviluppa così il controllo strategico, che ha lo scopo di accertare l'efficacia, a breve e a lungo, con cui si svolge l'attività e di verificare sia il conseguimento degli obiettivi e delle strategie sia l'andamento dei fattori interni ed esterni all'impresa per individuare minacce e opportunità che possono verificarsi. Sono molti i contributi sul controllo strategico (Lorange, 1980; Newman, 1981; Canziani, 1984; Amigoni, 1988; Coda, 1988; Paolini, 1993; Cavalieri, 1995), la maggior parte di essi cerca di capire se si tratta di un tipo di controllo distinto dal controllo di gestione. Bergamin (1991) propone un modello unico ed integrato. Ciò perché, il successo delle imprese deriva dalla formulazione della strategia ma anche dal processo

${ }^{2}$ Sulla logica prospettica, si veda Giannessi, Le aziende di produzione originaria, vol.I, Le aziende agricole, C. Cursi, Pisa, 1960; Caramiello, L'indagine prospettiva nel campo aziendale, C. Cursi, Pisa, 1965. 
decisionale che giorno dopo giorno porta alla sua realizzazione. Appare quindi necessario avere una visione unitaria del controllo in grado di bilanciare le esigenze di breve con quelle di lungo periodo.

In presenza di instabilità ambientale, la linearità del rapporto tra scelte di gestione e sistema di controllo mal si concilia anche con due tradizionali capisaldi concettuali, che richiedono un ripensamento: 1) il processo di decisione strategica è episodico e 2) la strategia è un elemento esogeno.

La strategia non è un processo episodico ma un processo di sviluppo continuo, nel quale le capacità strategiche sono incorporate nella gestione quotidiana (Langfield, Smith, 2007). Non è pertanto possibile "fermare" la strategia di un'impresa ad un dato istante per esaminare gli effetti sugli altri elementi del sistema organizzativo. Siamo convinti che la necessità di nuove strategie può emergere in qualsiasi momento della vita aziendale, sia nelle funzioni sia nei processi, come effetto del learning by doing.

La strategia non può neanche essere un elemento di contesto visto che $\mathrm{i}$ manager formulano decisioni strategiche senza soluzioni di continuità (Mintzberg, 1994). Pertanto, essa non può essere esterna al controllo ma dovrebbe interagire continuamente con lo stesso. Nel modello di controllo interattivo di Simons (1996) è infatti possibile osservare due "interventi" della gestione operativa su quella strategica. Il primo riguarda l'imprenditorialità diffusa e le iniziative strategiche ed operative che emergono in alcune aree dell'attività aziendale le quali influenzano i processi di formazione della strategia intenzionale; il secondo, più intenso, riguarda l'apprendimento e la revisione dei modelli mentali e dell'orientamento strategico di fondo e cerca di trasferire input innovativi nel sistema di idee del soggetto economico (Coda-Mollona, 2002).

Ne consegue che, soprattutto nell'ambiente instabile, non è proponibile la linearità del rapporto tra scelte di gestione e sistema di controllo di gestione. Si rende pertanto necessario il superamento della concezione degli studi classici che mettono in evidenza il carattere conoscitivo-razionale del processo di formulazione della strategia e di conseguenza distinguono questo momento da quello dell'attuazione della stessa.

Il primo contributo inserito in questo numero della rivista di Coller, Collini e Frigotto sembra dare una svolta significativa alla "lettura" del rapporto tra scelte di gestione e sistema di controllo. Attraverso l'analisi di un caso studio, il rapporto tra strategia e controllo è sviluppato superando la visione tradizionale, che pone il controllo in posizione subordinata rispetto alla strategia. Gli autori, partendo dall'idea che la strategia è invece un processo continuo che si interseca e interagisce con le decisioni quotidiane, dimostrano come il sistema di controllo partecipa alla realizzazione ma an- 
che alla formulazione della strategia. Viene così meno il carattere episodico che tradizionalmente è assegnato alla strategia, ma viene anche messa in evidenza la possibile bi-direzionalità della relazione strategia e controllo.

In senso più ampio ne deriva che, non sono solo le scelte di gestione a richiedere interventi di progettazione e ri-progettazione nei sistemi di controllo, ma potrebbero essere anche questi ultimi a consentire la costruzione di nuovi significati mettendo in luce emergenti problematiche gestionali.

Tra scelte di gestione e sistema di controllo di gestione si determina pertanto la seguente relazione:

scelte di gestione $\leftrightarrow$ sistema di controllo di gestione

La bidirezionalità di questa relazione ha degli effetti significativi: rende vana la ricerca, in un dato momento, della coerenza del sistema di controllo rispetto alla strategia; influenza la progettazione/riprogettazione del sistema di controllo perché la strategia si determina all'interno di una relazione dinamica che include anche il sistema di controllo.

Siamo di fronte ad una evoluzione della concezione degli studi classici sulla relazione tra gestione e controllo, che sicuramente si rifletterà su vari temi legati al controllo.

\section{Riflessi del rapporto tra scelte di gestione e sistema di controllo sulla pro- gettazione del sistema di misurazione delle performance}

Il sistema di misurazione delle performance è influenzato dal rapporto, appena esaminato, tra scelte di gestione e sistema di controllo, perché esso è un sub-sistema dello stesso sistema di controllo. Pertanto, l'evoluzione subita nel tempo dal sistema di misurazione deriva dai cambiamenti intervenuti nella "filosofia" aziendale, che hanno influenzano anche il sistema di controllo. È stato sinteticamente considerato il passaggio dalla "filosofia" aziendale rivolta alle dinamiche interne all'azienda alla "filosofia" rivolta all'esterno. Si esaminano brevemente gli effetti di tale passaggio sul sistema di misurazione.

In presenza di una cultura rivolta all'interno dell'azienda, l'interesse dell'imprenditore, si è già affermato, è rappresentato dal prodotto, dalla produzione, dalla "fabbrica" e quindi la variabile critica per perseguire vantaggi di costo è l'efficienza. Ne consegue che, il sistema di misurazione delle performance è progettato per accogliere misure economico-finanziarie (margini di profitto, variazioni di budget, fatturato, ROI). 
Così come avviene per il sistema di controllo, anche il sistema di misurazione si adegua alle necessità strategiche tese a perseguire il vantaggio competitivo.

Con l'apertura all'esterno dell'azienda, l'interesse dell'imprenditore è invece rivolto verso il cliente con la varietà e variabilità dei suoi bisogni, il mercato e l'ambiente esterno all'azienda. La qualità, il tempo e la flessibilità sono i nuovi fattori critici di successo che, assieme all'efficienza, permettono di conseguire il vantaggio competitivo. Pertanto di tali fattori è permeata la strategia che, attraverso il sistema di controllo, influenza il sistema di misurazione.

In questo contesto, i tradizionali sistemi di misurazione delle performance di natura economico-finanziaria presentono dei limiti largamente esaminati in letteratura (Johnson e Kaplan, 1987; Amigoni e Miolo Vitali, 2003; Ferraris Franceschi, 2007). Qui si fa riferimento solo ad un aspetto che influenza significativamente la progettazione del sistema di misurazione. Cioè alla circostanza che il successo dell'azienda è un concetto multidimensionale essendo collegato sia al conseguimento degli obiettivi economico-finanziari sia al modo in cui la stessa azienda si adatta all'ambiente in cui agisce (Coda, 1988). Ne consegue che, il sistema di misurazione dovrebbe avere una struttura multidimensionale, cioè accogliere misure quantitative e qualitative; misure finanziarie e non finanziarie.

In presenza di limiti, si tenta allora di sviluppare modelli di misurazione della performance aziendale in cui si cerca di individuare le variabili-causa e le variabili-effetto per capire i nessi che le collegano ed identificare le azioni convenienti per guidare le scelte della gestione. Ciò porta ad utilizzare nuove "lenti" di misurazione che determinano l'affermarsi del paradigma della multidimensionalità (Hendricks et al., 1998; Marchi, 2009; Castellano, 2011). In particolare, si riconosce che le misure non finanziarie pur avendo, per loro natura, un carattere più operativo, nella realtà assumono rilevanza tattica e strategica.

Sfruttando anche le potenzialità offerte dall'Information Technology, vengono pertanto sviluppati modelli concettuali globali per la rappresentazione delle performance aziendali. Si citano solo quelli più noti proposti in dottrina. Lo Strategic Measurement and Reporting Techique (SMART model), di Lynch e Cross (1991), si presenta in forma piramidale e prevede l'impiego di misure esterne ed interne di performance. Il modello di Fitzgerald et al. (1991) è costruito sul collegamento tra misure "risultato" (competitività e performance economico-finanziaria) e misure "determinative" (qualità, flessibilità, impiego di risorse ed innovazione). Il Macro Process Model, di Brown (1996), si focalizza su un processo di business caratteriz- 
zato da cinque fasi (inputs, processing systems, outputs, outcomes e goals) per ognuna delle quali prevede l'individuazione di specifiche misure. Viene così messo in evidenza il legame causale che esiste tra risorse (inputs) ed obiettivi strategici (goals). La Balanced Scorecard, di Kaplan-Norton (1996), il modello più noto, è uno strumento di controllo unico, si regge sul bilanciamento tra misure esterne ed interne e finanziarie e non finanziarie; è costruito sulla manifestazione dei nessi causali tra obiettivi strategici, attività, risorse e competenze; è sistematizzato in quattro dimensioni di performance.

L'evoluzione dei sistemi di misurazione delle performance appena accennata non modifica il legame tra lo stesso sistema di misurazione ed il rapporto tra scelte di gestione e sistema di controllo. Il sistema di misurazione è infatti sempre di supporto alle necessità della gestione aziendale.

Il legame con la gestione aziendale dovrebbe realizzarsi anche quando il sistema delle misurazioni viene applicato non alle performance, ma ai costi aziendali.

La suddetta problematica è affrontata nell'articolo di Rea e Stella con specifico riferimento alla misurazione dei costi ambientali, la cosiddetta Environmental Management Accounting (EMA).

Partendo dal quadro dottrinale dell'Activity-Based Costing nell'ambito dell'EMA, gli autori svolgono alcune considerazioni su una possibile combinazione dell'approccio Activity-Based alla prospettiva del ciclo di vita per la contabilizzazione dei costi ambientali d'impresa.

Le riflessioni presenti in questo contributo sono un valido aiuto per chi desidera esplorare il complesso tema della misurazione dei costi ambientali all'interno delle imprese.

Con riferimento al presente numero della rivista, i restanti tre contributi si occupano della progettazione di un sistema di misurazione delle performance e sembrano aderire alla tesi, consolidata in letteratura, del nesso causale tra gestione e sistema di controllo.

L'articolo di Rizza si occupa della progettazione di un sistema di misurazione delle performance negli ambiti interorganizzativi. Viene pertanto analizzato un sistema di misurazione che si estende oltre i confini dell'azienda, coerentemente all'affermarsi delle relazioni cooperative tra aziende.

Prendendo spunto dalla letteratura economico-aziendale sui sistemi di misurazione delle performance riferiti alla singola azienda, Rizza propone un modello che mette in evidenza come le finalità di una relazione interorganizzativa determinano specifici fabbisogni informativi che, a loro volta, 
richiedono misure di performance idonee a verificare il perseguimento delle predette finalità.

Per dare riscontro all'idea guida sviluppata in precedenza, l'autrice riporta un caso studio di un'azienda che impiega misure di performance interorganizzative differenziate, per ciascuna delle relazioni interorganizzative di cui è partner, in funzione degli scopi perseguiti dalla relazione.

Il modello proposto rappresenta una svolta significativa nella progettazione di un sistema di misurazione delle performance in ambiente interorganizzativo perché supera l'idea prevalente in letteratura secondo la quale tale sistema deve essere mirato solo agli aspetti tipici dei processi produttivo-distributivi frazionati tra più aziende partner. Il modello presentato porta invece a misure non necessariamente articolate su molteplici dimensioni, ma specifiche per l'ambito relazionale a cui si riferiscono e che derivano, di volta in volta, dalle finalità che caratterizzano ciascuna relazione interorganizzativa in un dato momento. Un modello così progettato non considera pertanto misure non necessarie, che potrebbero far distogliere l'attenzione dalle informazioni rilevanti ai fini della gestione della stessa relazione.

Gli ultimi due contributi della rivista si interessano infine della progettazione o riprogettazione dei sistemi di misurazione delle performance in specifici contesti settoriali, in particolare nelle istituzioni operanti nel settore culturale. Essi sono stati realizzati nell'ambito del Gruppo di Studio e Attenzione A.I.D.E.A su "Politiche e management del Patrimonio Culturale nelle diverse prospettive del valore. Strumenti e metodi di misurazione e valutazione", a cura di Fabio Donato e Barbara Sibilio. Entrambi i lavori, che presentano casi di istituzioni culturali, si propongono di indagare se $i$ sistemi di misurazione realmente operanti all'interno di tali istituzioni siano o meno coerenti rispetto a quanto proposto dalla letteratura economicoaziendale.

Il contributo di Nati esamina lo "stato" del sistema di misurazione del Museo Internazionale delle Ceramiche in Faenza. Tale sistema si caratterizza per la presenza di un dettagliato bilancio preventivo ed assestato, per lo sviluppo di un sistema di analisi dei costi e per l'impiego di indicatori di natura físico-tecnica. La ricerca svolta mette in evidenza come il sistema di misurazione adottato necessita di alcuni interventi di miglioramento per aumentarne la capacità informativa e renderlo più idoneo al conseguimento delle finalità a cui è diretto.

Il contributo di Marigonda esamina invece il sistema bibliotecario dell'Università IUAV di Venezia. L'analisi condotta è di tipo dinamico, infatti mette in evidenza il percorso di sviluppo del sistema di misurazione in tale istituzione. In questo caso, le tappe critiche sono rappresentate dal pas- 
saggio in corso verso un sistema di contabilità economico-patrimoniale, dalla necessità di un più diffuso utilizzo delle informazioni provenienti dal sistema di misurazione anche ai fini comunicativi esterni, dall'ideazione di un sistema di controllo di gestione finalizzato all'implementazione della strategia.

Tutti i contributi presentati in questo numero della rivista sulla progettazione del sistema di misurazione delle performance aderiscono alla tradizionale tesi del rapporto causale tra gestione e sistema di controllo. È auspicabile che i futuri lavori su questo tema accolgano la bi-direzionalità di tale relazione, così come è stata chiarita nel primo contributo di questo numero.

\section{Bibliografia}

Amigoni F., Miolo Vitali P., a cura di (2003), Misure multiple di performance, Milano, Egea.

Amigoni F. (1988), Misurazioni d'azienda. Programmazione e controllo, Milano, Giuffrè.

Andrews K.R. (1971), The concept of corporate strategy, Homewood, Dow Jones-Irwin.

Ansoff H.I., Declerck R.P., Hayes R.L. (1976), From Strategic Planning to Strategic Management, New York, Wiley \& Sons.

Anthony R.N. (1967), Sistemi di pianificazione e controllo, Milano, Etas.

Bergamin M. (1991), Programmazione e controllo in un'ottica strategica, Torino, Utet.

Brown M.G. (1996), Keeping score: Using the right metrics to drive world class performance, New York, Quality Resources.

Brunetti G. (1979), Il controllo di gestione in condizioni ambientali perturbate, Milano, FrancoAngeli.

Brusa L., Zambrogna L. (1991), Pianificazione e controllo di gestione. Creazione del valore, cost accounting e reporting direzionale: tendenze evolutive, Milano, EtasLibri.

Canziano A. (1984), La strategia aziendale, Milano, Giuffrè.

Castellano N. (2011), Modelli e misure di performance aziendale: analisi della letteratura e spunti di ricerca, Management Control, 1.

Cavalieri E. (1995), Variabilità e strutture d'impresa, Padova, Cedam.

Coda V., Mollona E. (2002), Il governo della dinamica della strategia, Finanza, Marketing e Produzione, 4.

Coda V. (1988), L'orientamento strategico dell'impresa, Torino, Utet.

Eminente G. (1986), Pianificazione e gestione strategica dell'impresa, Bologna, il Mulino.

Ferraris Franceschi R. (2007), La natura del sistema manageriale di pianificazione e controllo, in Ferraris Franceschi R. (a cura di), Pianificazione e Controllo, Giappichelli, Torino.

Fitzgerald L., Johnston R., Brignall T.J., Silvestro R., Voss C. (1991), Performance measurement in service businesses, London, The chartered Institute of Management Accountants.

Hendricks J.A., Defreitas D.G., Walker D.K. (1998), Ridisegnare il sistema di misurazione delle performance in logica di empowerment: l'esperienza Caterpillar, Budget, 16. 
Johson H.T., Kaplan R.S. (1987), Relevance lost: the rise and fall of management accounting, Boston, Harvard Business School Press.

Kaplan R.S., Norton D.P. (1996), Balanced Scorecard. Tradurre la strategia in azione, Torino, Isedi.

Langfield-Smith K. (2007), A review of quantitative research in management control system and strategy, in Chapman C.S., Hopwood A.G., Shields M.D., a cura di, Handbook of Management Accounting Research, Amsterdam, Elsevier.

Lorange P. (1980), Pianificazione strategica, Milano, McGraw Hill.

Lynch R.L., Cross K.F. (1991), Measures up - The essential guide to measuring business performance, London, Mandarin.

Marchi L. (2009), Il processo di controllo, in Marasca S., Marchi L., Riccaboni A. (a cura di), Controllo di gestione. Metodologie e strumenti, Arezzo, Knowita.

Mintzberg H. (1994), Ascesa e decline della pianificazione strategica, Milano, Isedi.

Newman W.H. (1981), Direzione e sistemi di controllo, Milano, EtasLibri.

Paolini A. (1993), Il controllo strategico, uno schema d'analisi, Milano, Giuffrè.

Scott M.C. (1998), Value Drivers. The manager's guide to driving corporate value creation, New York, Wiley \& Sons.

Simons R. (1996), Leve di controllo:i nuovi sistemi per guidare le imprese, Milano, FrancoAngeli. 